\title{
Optimal conditions for a control problem associated to an economical model
}

\author{
Olivia BUNDĂU ${ }^{1, *}$, Adina JURATONI ${ }^{1, * *}$ \\ ${ }^{1}$ Departament of Mathematics,"Politehnica"University of Timisoara, P-ta Victoriei, 2, Timisoara, \\ 300006, Romania
}

\begin{abstract}
In this paper, we presents a growth model in infinite and continuous time, leads to a control optimal problem. Using the Pontryagin's principle we done necessary conditions for optimality. Also, we prove the existence, uniqueness and stability of the steady state for a differential equations system.
\end{abstract}

\section{Introduction}

In this paper we consider a version of the Ramsey growth model in infinite and continuous time ([3]). In this model, the consumption and, hence, the saving rate are determined by optimizing households and firm that interact on competitive market. In this economy, the consumer chooses at each moment in time the level of consumption so as to maximize the global utility on the infinite time, given by exponential function. This economical growth model of Ramsey leads to an optimal control problem. We formulate a theorem which gives the necessary conditions for a control function to solve our optimal control problem. An analogous theorem is formulate by Ş. Cruceanu and C. Vîrsan ([4]). We show that the optimal solution of the optimal control problem is the solution of a system of differential equations, which exhibits saddle-path stability. An analogous result is derived by Barro and Sala-iMartin for another version of the Ramsey model ([1]).

The outline of this paper is as follows. In section 2, we present the model. In Section 3, we give the necessary conditions for the optimal solution of the optimal growth problem in this model. In Section 4, we determine the steady state of the optimal growth problem and we show that it is a saddle point. In Section 5, the conclusions are discussed.

\section{The model}

We consider an economical model that contains a household and a firm. Also, we assume the economy is closed with competitive market, all of the stock capital must be owned by someone in economy and the net foreign debt is zero.

The firm produces goods, pays wages for labor, and makes rental payments for capital. Output is determined by the following production function

$$
Y=F(K, L)
$$

\footnotetext{
*e-mail: olivia.bundau@upt.ro

**e-mail: adina.juratoni@upt.ro
} 
where $K$ is capital, $L$ is labor force, $F: \mathbb{R}_{+} \times \mathbb{R}_{+} \rightarrow \mathbb{R}_{+}$is a function of class $C^{2}$ having the following properties

$$
\begin{gathered}
\frac{\partial F(K, L)}{\partial K}>0, \frac{\partial F(K, L)}{\partial L}>0,(\forall) K>0, L>0 ; \\
\frac{\partial^{2} F(K, L)}{\partial K^{2}}<0, \frac{\partial^{2} F(K, L)}{\partial L^{2}}<0,(\forall) K>0, L>0 ; \\
F(\lambda K, \lambda L)=\lambda F(K, L)=\lambda Y,(\forall) \lambda>0 ; \\
\lim _{K \rightarrow 0} \frac{\partial F(K, L)}{\partial K}=\lim _{L \rightarrow 0} \frac{\partial F(K, L)}{\partial L}=\infty, \lim _{K \rightarrow \infty} \frac{\partial F(K, L)}{\partial K}=\lim _{L \rightarrow \infty} \frac{\partial F(K, L)}{\partial L}=0, \\
F(K, 0)=F(0, L)=0 .
\end{gathered}
$$

The firm seeks to maximize the present value of profits, given by

$$
\text { Profit }=F(K(t), L(t))-(r(t)+\delta) K(t)-w(t) L(t),
$$

where $r(t)$ and $w(t)$ are given, $r$ is the rental rate of capital, $w$ is wage per worker, and $\delta$ is depreciation of capital.

We define

$$
\begin{aligned}
& k(t)=\frac{K(t)}{L(t)}-\text { the capital per worker; } \\
& y(t)=\frac{Y(t)}{L(t)}-\text { the output per worker. }
\end{aligned}
$$

Using the homogeneity condition we have

$Y=F(K, L)=L \cdot F\left(\frac{K}{L}, 1\right)=L \cdot f(k)$, where $f(k)=F(k, 1)$.

Then, the output per worker is

$$
y=f(k)
$$

Using the properties of function $F$ we obtain the properties of function of class $C^{2}, f$ : $f(0)=0 ; f^{\prime}(k)>0, f^{\prime \prime}(k)<0(\forall) k>0 ; \lim _{k \rightarrow 0} f^{\prime}(k)=\infty ; \lim _{k \rightarrow \infty} f^{\prime}(k)=0$.

Profit for this firm can be written as

$$
\text { Profit }=L(t)(f(k(t))-(r(t)+\delta) k(t)-w(t))
$$

On a competitive market, the economical condition for the maximum profit, that could be obtained by the firm to be zero is given by

$$
\begin{aligned}
f^{\prime}(k) & =r+\delta \\
f(k)-k f^{\prime}(k) & =w .
\end{aligned}
$$

In this economy the consumer chooses at each moment in time the level of consumption $c(t)$ such that to maximize the global utility, given by

$$
U=-\frac{1}{\theta} \int_{0}^{\infty} e^{-(\rho-n) t} e^{-\theta c(t)} d t
$$


subject to the budget constraint for the household, given in ([1]) by

$$
\dot{K}(t)=r(t) K(t)+w(t) L(t)-C(t) .
$$

We denoted $c$ consumption, $\rho>0$ is a discount rate and $\theta>0$.

The size of the household grows at rate $n$.

The budget constraint for the household can be written in intensive form thus

$$
\dot{k}(t)=r(t) k(t)+w(t)-c(t)-n k(t)
$$

where $c(t)=\frac{C(t)}{L(t)}$ is the consumption per capita.

Initial stock of capital that is available for household is $K_{0}$. Thus, initial stock of capital per capita is $k_{0}$.

\section{Determination of optimality conditions}

The economical problem of choice in every moment $t$ of the size of consumption so as to maximize the global utility taking into account to the budget constraint for the household and the initial stock of capital $k_{0}$ leads us to the following mathematical optimization problem $(P)$ :

The problem $\mathbf{P}$. To determine $\left(k^{*}, c^{*}\right)$ which maximize the following functional

$$
-\frac{1}{\theta} \int_{0}^{\infty} e^{-(\rho-n) t} e^{-\theta c(t)} d t
$$

in the class of functions $k \in A C\left([0, \infty), \mathbb{R}_{+}\right), c \in \mathcal{X}=\{c:[0, \infty) \rightarrow[0, A], c-$ measurable $A<\infty\}$ which verifies:

$$
\begin{array}{ll}
\dot{k}(t)= & r(t) k(t)+w(t)-c(t)-n k(t), \text { a.e. } t \in[0, \infty) \\
k(0)= & k_{0} .
\end{array}
$$

In our problem $\mathbf{P}, k$ is a state variable and $c$ is a control variable.

We will continue to determine the necessary conditions for optimality problem $P$. To do this we will apply the maximum principle of Pontryagin. For this we define ( as in [1], [2]) the function of Hamilton-Pontriaghin $H:[0, \infty) \times[0, A] \times \mathbb{R} \times[0, \infty) \rightarrow \mathbb{R}$ given by

$$
H(k, c, \mu, t)=-\frac{1}{\theta} e^{-(\rho-n) t} e^{-\theta c}+\mu(r k+w-c-n k)
$$

Theorem 3.1. Let $\left(k^{*}(t), c^{*}(t)\right)$ be an optimal solution which solves problem $\mathbf{P}$. Then there exist an absolutely continuous function $q(t)$ such that for all $t$

$$
\begin{aligned}
& \dot{q}(t)=q(t)(\rho-r(t)) \\
& q(t)=e^{-\theta c^{*}(t)}
\end{aligned}
$$

Proof. Let $\left(k^{*}(t), c^{*}(t)\right)$ an optimal solution for $\mathbf{P}$.

The Hamiltonian function associated to the problem $(P)$ is

$$
H(k(t), c(t), \mu(t), t)=-\frac{1}{\theta} e^{-(\rho-n) t} e^{-\theta c(t)}+\mu(t)(r(t) k(t)+w(t)-c(t)-n k(t))
$$


From the Pontryaghin's principle there is an absolutely continuous function $\mu(t)$ such that

$$
\dot{\mu}(t)=-\frac{\partial H}{\partial k}=-\mu(t)(r(t)-n)
$$

and $c^{*}(t)$ is value $c \in[0, A]$ which maximize

$$
H\left(k^{*}(t), c, \mu(t), t\right)=-\frac{1}{\theta} e^{-(\rho-n) t} e^{-\theta c(t)}+\mu(t)\left(r(t) k^{*}(t)+w(t)-c-n k^{*}(t)\right)
$$

Using the transformation $\mu(t)=e^{-(\rho-n) t} q(t)$, the Hamilton function associated to $\mathbf{P}$ becomes

$$
\begin{aligned}
H(k(t), c(t), q(t), t)= & e^{-(\rho-n) t}\left\{-\frac{1}{\theta} e^{-\theta c(t)}+\right. \\
& +q(t)(r(t) k(t)+w(t)-c(t)-n k(t))\}
\end{aligned}
$$

and (3.5) becomes

$$
H\left(k^{*}(t), c, q(t), t\right)=e^{-(\rho-n) t}\left\{-\frac{1}{\theta} e^{-\theta c}+q(t)\left(r(t) k^{*}(t)+w(t)-c-n k(t)\right)\right\} .
$$

The first and second derivatives of function $H$ with respect to $c$ are

$$
\begin{gathered}
H_{c}^{\prime}\left(k^{*}(t), c, q(t), t\right)=e^{-(\rho-n) t}\left(e^{-\theta c}-q(t)\right) \\
H_{c c}^{\prime \prime}\left(k^{*}(t), c, q(t), t\right)=-\theta e^{-(\rho-n) t} e^{-\theta c} .
\end{gathered}
$$

and we obtain that $H$ is a concave function of $c$.

Because $c^{*}(t)$ is that $c \in[0, A]$ which maximizes (3.6) and $H$ is a concave function of $c$ we have

$$
H_{c}^{\prime}\left(k^{*}(t), c^{*}(t), q(t), t\right)=0
$$

thus, we obtain

$$
q(t)=e^{-\theta c^{*}(t)}
$$

We using again the transformation $\mu(t)=e^{-(\rho-n) t} q(t)$, (3.4) becomes

$$
\dot{q}(t)=q(t)(\rho-r(t))
$$

Remark 1. The optimal trajectory of the problem $(\mathbf{P})$ is a solution of the following system of differential equations

$$
\begin{aligned}
& \dot{k}(t)=r(t) k(t)+w(t)-c(t)-n k(t) \\
& \dot{c}(t)=-\frac{1}{\theta}(\rho-r(t))
\end{aligned}
$$

Remark 2. The optimal trajectory of the problem $(\mathbf{P})$ in the conditions of a competitive equilibrium is the solution of the following system

$$
\begin{aligned}
\dot{k}(t) & =f(k(t))-(n+\delta) k(t)-c(t) \\
\dot{c}(t) & =-\frac{1}{\theta}\left[\rho-\left(f^{\prime}(k(t))-\delta\right)\right]
\end{aligned}
$$




\section{Qualitative analysis of the optimal solution}

\section{Proposition 4.1. The system of differential equations}

$$
\begin{aligned}
\dot{k}(t) & =f(k(t))-(n+\delta) k(t)-c(t) \\
\dot{c}(t) & =-\frac{1}{\theta}\left[\rho-\left(f^{\prime}(k(t))-\delta\right)\right]
\end{aligned}
$$

exhibits saddle-path stability.

Proof. For to determine the steady state of the above system we choose the stationary solutions $k(t)=k^{*}, c(t)=c^{*}$. From $\dot{c}(t)=0$ we obtain

$$
f^{\prime}\left(k^{*}\right)=\rho+\delta .
$$

Using the properties of the function $f$ result that the equation (4.2) has a unique solution $k=k^{*}$ with which we may determine

$$
c^{*}=f\left(k^{*}\right)-(n+\delta) k^{*}
$$

The point $\left(k^{*}, c^{*}\right)$ represents a steady state for the system of differential equations.

To investigate the stability of the steady state $\left(k^{*}, c^{*}\right)$ we linearize the system (4.1) around the study state and we obtain

$$
\begin{aligned}
& \dot{k}(t) \approx\left(f^{\prime}\left(k^{*}\right)-n-\delta\right)\left(k(t)-k^{*}\right)-\left(c(t)-c^{*}\right) \\
& \dot{c}(t) \approx \frac{1}{\theta} f^{\prime \prime}\left(k^{*}\right)\left(k(t)-k^{*}\right)
\end{aligned}
$$

The matrix of the linearized system is $\left(\begin{array}{cc}f^{\prime}\left(k^{*}\right)-n-\delta & -1 \\ \frac{1}{\theta} f^{\prime \prime}\left(k^{*}\right) & 0\end{array}\right)$.

The eigenvalues are solutions of equation

$$
\lambda^{2}-\lambda\left(f^{\prime}\left(k^{*}\right)-n-\delta\right)+\frac{1}{\theta} f^{\prime \prime}\left(k^{*}\right)=0 .
$$

Because $f$ is a concave function, it results that the determinant of the equation is positive.

$$
\Delta=\left(f^{\prime}\left(k^{*}\right)-n-\delta\right)^{2}-4 \frac{1}{\theta} f^{\prime \prime}\left(k^{*}\right)>0
$$

and

$$
p=\frac{1}{\theta} f^{\prime \prime}\left(k^{*}\right)<0
$$

Hence, the equation (4.5) has two real roots for the contrary signs

$$
\lambda_{1,2}=\frac{\left(f^{\prime}\left(k^{*}\right)-n-\delta\right) \pm \sqrt{\Delta}}{2} .
$$

Using (4.2), it results that the relation (4.6) which gives the eigenvalues, can be written thus

$$
\lambda_{1,2}=\frac{\rho \pm \sqrt{\Delta}}{2}
$$

The eigenvalues of the differential system (4.4) being the real numbers with contrary signs, it results that the steady state $\left(k^{*}, c^{*}\right)$ is the saddle point. 


\section{Conclusions}

In this paper, we analyzed a growth economical model in which the utility is given by exponential function. We described the behavior of the consumption and capital when the utility function is an exponential function. The analysis reveals that there is a unique steady state of the optimal growth problem in this model. This steady state is a saddle point.

\section{References}

[1] R. J. Barro, X. Sala-i-Martin, Economic Growth, New York. McGraw-Hill (1995).

[2] O. Bundău, M. Neamţu, Optimal conditions for the control problem associated to a Ramsey model, Sci. Bull. of the "Politehnica" University of Timisoara, 2008.

[3] F. P. Ramsey, A Mathematical Theory of Saving, The Economic Jurnal, Vol.38,No. 152, 1928, pp. 543-559

[4] Ş. Cruceanu, C. Vîrsan, Elemente de Control optimal şi Aplicaţii in Economie, Ed. Teh. Bucarest, 1978. 